\title{
Computed Tomographic Findings of Abdominal Granulomas in Three Miniature Dachshund Dogs
}

\author{
Takeshi Tsuka*, Yoshiharu Okamoto, Tomohiro Osaki, Takehito Morita, Yuji Sunden, Kazuo Azuma, Masamichi Yamashita, Norihiko Ito, \\ Yusuke Murahata and Tomohiro Imagawa
}

Department of Veterinary Clinical Medicine, School of Veterinary Medicine, Faculty of Agriculture, Tottori University, Tottori, Japan

*Corresponding author: Takeshi Tsuka, Veterinary diagnostic imaging, Department of Veterinary Clinical Medicine, School of Veterinary Medicine, Faculty of Agriculture, Tottori University, 4-101, Koyama-Minami, Tottori, Japan, Tel: +81-857-31-5435; Fax: +81-857-31-5449; E-mail: tsuka@muses.tottori-u.ac.jp

Received: 28 Dec, 2018 | Accepted: 30 Jan, 2019 | Published: 06 Feb, 2019

Citation: Tsuka T, Okamoto Y, Osaki T, Morita T, Sunden Y, et al. (2019) Computed Tomographic Findings of Abdominal Granulomas in Three Miniature Dachshund Dogs. J Anim Sci Res 3(1): doi dx.doi.org/10.16966/2576-6457.122

Copyright: (C) 2019 Tsuka T, et al. This is an open-access article distributed under the terms of the Creative Commons Attribution License, which permits unrestricted use, distribution, and reproduction in any medium, provided the original author and source are credited.

\begin{abstract}
Three miniature dachshund dogs were presented with chronic gastrointestinal upset including vomiting and diarrhea. In these patients, abdominal masses were detected on abdominal palpation. Computed tomography revealed that two and one patients had, respectively, multiple and solo formations of abdominal masses demonstrated as follows: (1) irregular margin of the mass (2) uniformity of contrast enhancement over all areas of the mass; (3) and maintenance of small intestinal lumen even if embedded by the mass. In all three patients, these abdominal masses were removed by laparotomy, and were pathologically diagnosed as a granuloma. These specific findings on computed tomographic images of abdominal granuloma will be useful for the diagnosis.
\end{abstract}

Keywords: Abdomen; Computed tomography; Granuloma; Miniature dachshund; Small intestine

\section{Introduction}

Abdominal granuloma is a proliferative disorder secondary to injury, infection, intestinal perforation, and inflammatory reactions due to various foreign materials [1]. The most commonly reported foreign materials include sutures (especially non-absorbable sutures) and gauze sponges retained after surgery, and are called stitch or suture granulomas and gossypibomas, respectively [1-4]. There has been an increase in the number of cases of abdominal granulomas caused by retained foreign materials in small animal practice $[1,4-$ 6]. This recently increased incidence of suture granuloma may be due to the high number of affected miniature dachshunds reported in Japan [7]. The relevant radiographic and ultrasonographic findings of such lesions have also been studied in small animals [1,4-6]. Gossypibomas are characterized by whirl-like gas patterns within a mass on radiographic images and a well-demarcated hypoechoic mass with highly echogenic, shadowing components on ultrasonographic views $[1,6]$. Suture granulomas may be diagnosed by homogenous soft tissue opacity using radiography, and have been observed with variety of ultrasound findings such as a capsule-like mass with slight acoustic shadowing, and a hyperechoic mass without acoustic shadowing [4,6]. However, clinical applications of computed tomography (CT) to small animals with abdominal granulomas have not been well discussed.

\section{Case Presentation}

Case 1 was a 3 -year-old spayed miniature dachshund dog presented for chronic vomiting for 3 months. There was no previous intra-abdominal surgery except for ovariohysterectomy. A mass was palpated in the cranial and middle region of the abdomen. Red blood cell counts decreased from $894 \times 10^{4}$ to $535 \times 10^{4} \mathrm{cells} / \mu \mathrm{l}$ during 1-week interval from first clinic. Leukocytosis was continued (white blood cell counts ranged 26800 to 57200 cells/ $\mu$ l). The use of radiography (Regius model 110; Konica Minolta Inc., Tokyo, Japan) showed multiple soft tissue opacity masses within both the left and right caudal lung lobes on the thoracic images. On abdominal radiographic views, a large mass was recognized on the caudal aspect of the gas-filled stomach (the gastric axis was caudally dislocated due to hepatomegaly), and it reached the region cranial to the bladder. A small amount of gas was observed within the lumen of the small intestine. The use of ultrasonography (HI VISION Preirus; HitachiAloka Medical, Ltd., Tokyo, Japan) showed severe expansion of the gallbladder with slight extension of the bile duct. The mass was seen as a mosaic composed of low and high echogenic structures in the left and middle regions of the abdomen (Figure 1A). Slight acoustic shadowing was observed deep to the abdominal mass. The animal was examined using an examined by a single-slice, slip-ring CT scanner (Pronto SE; Hitachi medical Co, Tokyo, Japan) under deep 
anesthesia. On the abdominal CT, the abdominal mass appeared to be derived from gastric wall, because the border between the gastric structure and the mass was unclear (Figure 1B). The margin of the mass was irregular. The mass had a length of $56.0 \mathrm{~mm}$, width of 56.4 $\mathrm{mm}$, and height of $87.5 \mathrm{~mm}$ (the sizes were shown in that order on subsequent images). Intravenous injection of contrast agent revealed heterogeneous contrast enhancement of the mass; the Hounsfield units (HUs) increased from 42 to 105 . There were 6 pulmonary masses, ranging in size from $6.0 \mathrm{~mm}$ to $16.0 \mathrm{~mm}$ in the longest diameter, within both the right and left caudal lobes. Examination of cytologic specimens obtained from the mass itself via ultrasonography-guided fine-needle aspiration revealed monometric accumulations of round nuclear cells, sometimes characterized as giant cells, together with cellular components consisting of neutrophils and fibrous cells (Figure 1C). Multiple formations of the masses into the thoracic and abdominal cavity on CT and accumulation of tumor-like giant cells in the cytology led to difficulty in the differential diagnosis between the abdominal granuloma and tumor before surgery. On laparotomy, the spongiform mass appeared to be derived from the caudal margin of the stomach, with the pylorus being most severely affected. The mass extended to the ventral region of both kidneys, and there were adhesions between the spleen and the caudal edge of the mass. A part of the small intestine was completely covered inside the mass. There were also adhesions between the mass and the hepatic lobes and the bile duct, resulting in bile thrombus. Gastrectomy was performed to include a large area of the caudal gastric wall near the pylorus. Blunt dissection of the mass from the liver, spleen, and small intestine was easily performed. However, the embedded small intestine developed a large discolored change, despite no destruction of the lumen. Therefore, the discolored region was completely removed and followed by an end-to-end anastomosis. Cholangioenterostomy was performed for the bile thrombus. However, the dog died by sudden cardiac arrest during surgery, possibly due to severe surgical invasiveness and the extended surgical time. A postmortem examination was declined by the owners. In the histopathology of the removed mass including a part of the gastric wall, the inflammatory cells (such as neutrophils and macrophages) were mainly accumulated within overall of the mass.
The gastric wall included slight degrees of infiltrations of inflammatory cells. Mitotic figure was not evident.

Case 2 was an 8-year-old miniature dachshund dog presented for chronic vomiting. At the age of 2 years, an ovariohysterectomy was performed. A mass could be palpated in the center of the abdomen. There were no hematological abnormalities; the white blood cell count was $12400 / \mu \mathrm{l}$. The $\mathrm{C}$ reactive protein (CRP) level was $>20 \mathrm{mg}$ / $\mathrm{dl}$ (the upper border for measurement) (cut off value: $1.0 \mathrm{mg} / \mathrm{dl}$ ). On abdominal radiography, the stomach was distended with gas. A soft tissue opacity mass was visualized caudal to the gas-filled stomach. On ultrasonography, a heterogeneous echogenic mass was seen near the enlarged spleen. The mass showed no shadowing. The CT examination revealed 4 masses in the abdominal cavity: one was close to the gastric cardia and was $15.0 \mathrm{~mm} \times 17.6 \mathrm{~mm} \times 14.3 \mathrm{~mm}$ in size; another mass close to the duodenum was $21.0 \mathrm{~mm} \times 18.2 \mathrm{~mm} \times 21.6 \mathrm{~mm}$ in size; the third mass was located within the space between the stomach and the spleen, and was $27.6 \mathrm{~mm} \times 30.3 \mathrm{~mm} \times 23.9 \mathrm{~mm}$ in size; and the fourth mass was located on the right side of the enlarged spleen and close to the right abdominal wall, and was $31.8 \mathrm{~mm} \times 33.9 \mathrm{~mm}$ $\times 33.9 \mathrm{~mm}$ in size (Figure 2A). Within this mass, the small intestine was embedded. The embedded region of the small intestine running through the mass maintained a lumen structure. All margins of the four masses were irregular. With contrast, the HUs increased over all areas of the four masses from the pre-contrast levels of 37.8 to 57.0 to post-contrast levels of 113.9 to 126.7. Cytologic specimens obtained from the third mass via CT-guided fine-needle aspiration revealed accumulations of inflammatory cells composed predominantly of neutrophils and macrophages (Figure 2B). Based on the CT findings showing embedding of the small intestine within the mass, abdominal granuloma could be suspected before surgery. On laparotomy, the four masses were bound to the adjacent organs due to accumulation of fibrous structures within each region of the abdominal cavity. However, the attachments were easily released with blunt dissection. The mass on the right side of the spleen was removed with the embedded region of the small intestine, and an end-to-end anastomosis was performed. The section of the removed mass appeared spongiform, with the
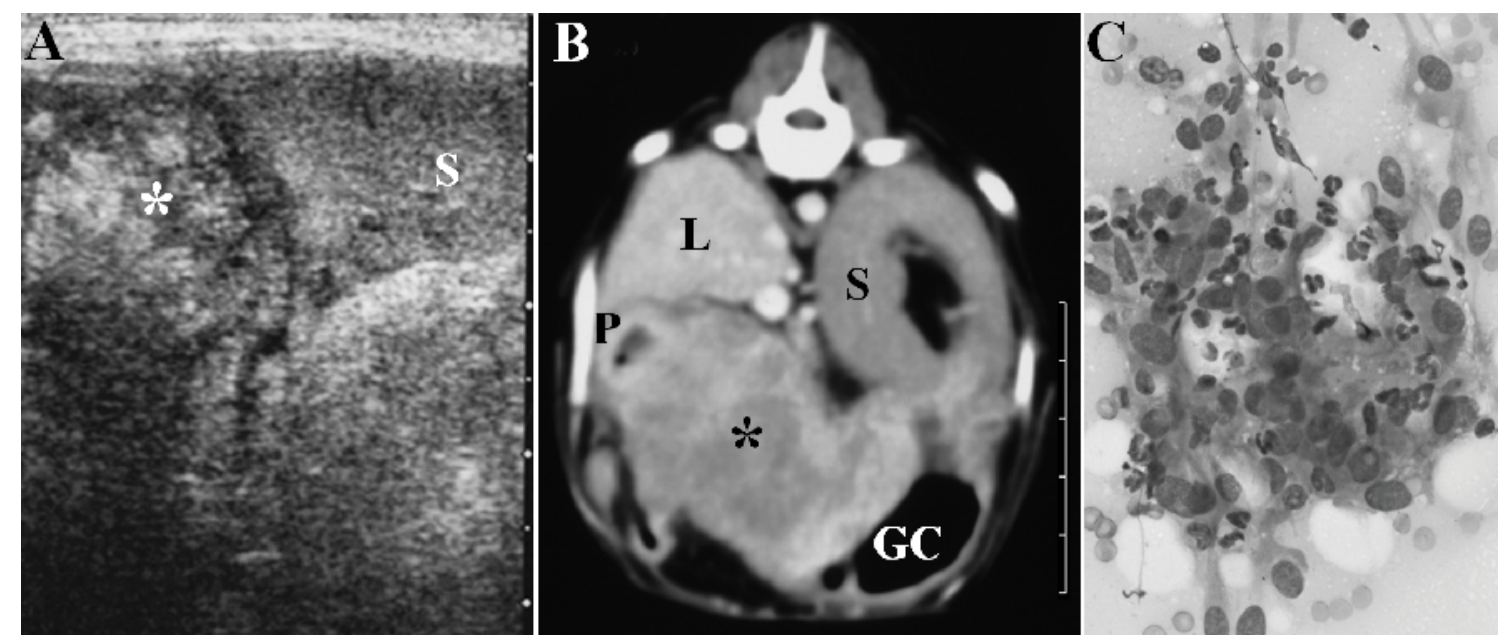

Figure 1: Ultrasonogoraphic (A) and CT images (B), and cytologic finding (C) of the abdominal granuloma in dog 1. (A) On the sagittal ultrasonographic image with a 6.5-MHz linear probe, the mass showed a mosaic echogenic structure (asterisk) and slight acoustic shadowing. S: Spleen. (B) On transverse abdominal CT image (window level: 40 , window width: 300), the mass showed a heterogeneous contrast-enhanced structure (asterisk) originating from the gastric wall and covering almost the entire area of the stomach. GC: Greater curvature of stomach. L: Liver. P: Pylorus. S: Spleen. A bar was shown $10 \mathrm{~mm}$. (C) Cytologic examination showed monometric accumulations of round nuclear cells together with cellular components consisting of neutrophils and fibrous cells. 
included small intestine maintaining its lumen (Figure 2C). Although intact removal of the masses within the space between the stomach and spleen and close to the duodenum was easy, intact removal of the mass close to the gastric cardia was impossible because of difficulty in the surgical approach. Long-term administration of prednisolone and antibiotics was performed postoperatively. The dog died 1 month after surgery from mechanical obstruction due to enlargement of the mass close to the gastric cardia. The histopathological finding of the removed mass included severe accumulations of inflammatory cells (such as neutrophils and macrophages). Lumen structures of the embedded intestine were maintained, and had slight degrees of infiltrations of inflammatory cells.

Case 3 was a 5-year-old miniature dachshund dog having a 1-year history of chronic diarrhea. This dog had undergone abdominal surgery to remove an abdominal testicle at 1 year of age. A mass could be palpated in the center to right region of the abdomen. There were no hematological abnormalities; the white blood cell count was 12000 cells/ $\mu$ l. The CRP level was $>20 \mathrm{mg} / \mathrm{dl}$. On lateral abdominal radiography, the round mass was visualized near the spleen, close to the abdominal wall. Gas fully filled the lumen of the small intestine. On the right-sided ultrasonographic view, the mass was seen as homogenously echogenic without acoustic shadowing at the lateral site of the duodenum. It was separated from the adjacent organs, including the liver and pancreas. On transverse CT images at the kidney level, the mass occupied largely the right-side and was located in the ventral region of the right kidney (Figure 3A). Its dimensions were $35.6 \mathrm{~mm} \times$ $29.5 \mathrm{~mm} \times 34.3 \mathrm{~mm}$. Ball-like accumulation of the small intestine was recognized surrounding the mass acting as a core. The small intestine meandered along the mass, and a part was embedded by the mass. The embedded region of the small intestine maintained the lumen without infiltration and destruction by the mass. The entire margin of the mass was irregular. Contrast enhancement was seen uniformly within all areas of the mass; the HUs increased from 53.9 before administration of the contrast agent to 160.2 after contrast medium injection. Based on the CT findings showing ball-like accumulation of the small intestine within the mass, abdominal granuloma could be suspected before surgery. On laparotomy, a fibrous structure allowing a constricting band into the space between the small intestine and the spongiform mass was seen (Figure 3B). The adhesion was easily broken down by blunt removal and dissection, and the small intestine could be separated from the mass. The embedded structure of the small intestine showed severe discolored change. Therefore, the abnormal region was removed, and an end-to-end anastomosis was performed. Prednisolone continued to be administrated over 2 months postoperatively. This dog was alive without recurrence after surgery. The histopathological finding of the removed mass included severe accumulations of inflammatory cells. Lumen structures of the embedded intestine were maintained, and had slight degrees of infiltrations of inflammatory cells.

\section{Discussion}

Abdominal granulomas generally occur secondary to injury, infection, intestinal perforation, and inflammatory reactions due to various foreign materials, including sutures (called stitch or suture granulomas) and surgical sponges (called gossypibomas) [1]. Possible initial causes were gastric perforation in Case 1 and inflammatory reactions secondary to retained suture in Case 2 and Case 3. Almost all of the surgical procedures require ligations using suture materials. Retained suture is a well-known cause of delayed surgical complications $[6,8,9]$. These complications usually develop due to a chronic inflammatory reaction surrounding ligatures and contamination during or after surgery, and abscesses or granulomas commonly occur [8]. Most of the clinical signs appear over several months and sometimes up to 2 years after surgery [1,5,8]. Local formations of granulomas (e.g., uterine or ovarian stump granulomas) or granulation developing relatively soon (e.g., months) after surgery appear to be associated with infiltration of the mucous membranes with infectious organisms $[1,6,10]$. On the other hand, the long interval of many years prior to the onset of clinical signs suggests that the primary cause of such lesions may not be an infection [8], but a slow immune response to an inert foreign material [5,9]. This process explains the multiple lesions seen in Case 1 and Case 2. In the present case series, all of the affected animals were miniature dachshunds. The miniature dachshund has been frequently listed as affected in Japan, despite the

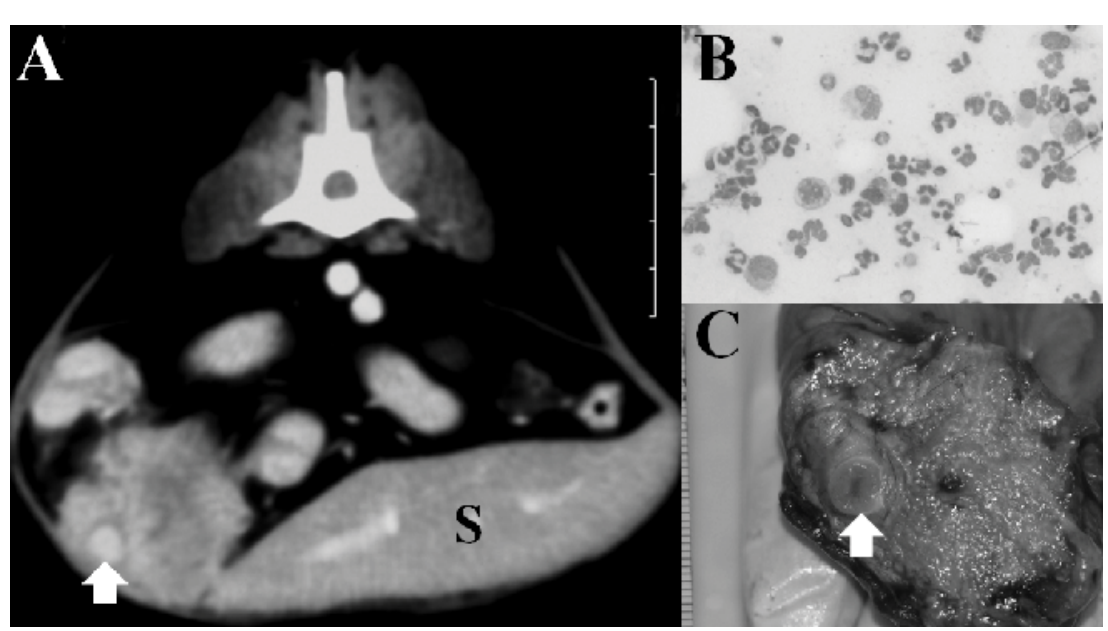

Figure 2: CT image (A), cytologic findings (B), and gross appearance (C) of the abdominal granulomas in dog 2. (A) Transverse abdominal CT view (window level: 40, window width: 300) shows the abdominal mass located on the right side of the enlarged spleen (S) and embedding the small intestine (arrow). A bar was shown $10 \mathrm{~mm}$. (B) Cytologic examination shows predominant accumulations of inflammatory cells composed of neutrophils and macrophages. (C) Gross section of the removed mass with an appearance identical to the CT findings of the mass in Figure 2A. Note the embedded small intestine which maintains its lumen (arrow). 


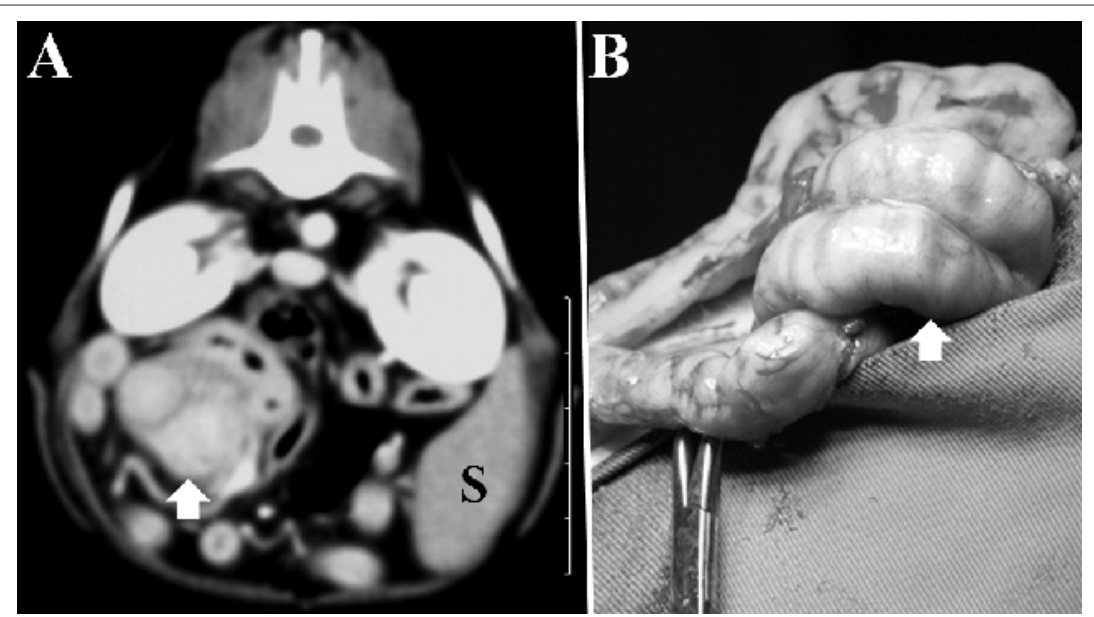

Figure 3: CT image (A) and intraoperative view (B) of the abdominal granuloma in dog 3. (A) Transverse abdominal CT view (window level: 60, window width: 300) shows a ball-like accumulation of the small intestine surrounding the mass. A part of the small intestine was embedded by the mass (arrow). (B) Intraoperative view shows coil-like adhesion of the small intestine due to proliferation of a fibrous structure into the space between the loops of small intestine.

fact that less breed specificity for the occurrence of such lesions has been reported worldwide [1,5,9]. Miniature dachshunds are known to be predisposed to pyogranulomatous inflammation in any adipose tissue $[7,11]$. Such lesions are considered to be part of an immunemediated disorder, because they have a good to excellent response to glucocorticoids and other immunomodulating drugs $[5,11]$.

In such lesions, abdominal pain was not a frequent clinical sign, as in our cases [1,2]. Many dogs had a palpable abdominal mass [1,6]. On blood examinations, an increased white blood cell count and neutrophilia were not always common hematological findings [1,2,6,7]. An increased CRP level may be useful for detection of granulomatous lesions, because CRP may be a highly sensitive indicator of systemic inflammation, resulting in occurrence of abdominal granulomas in miniature dachshunds $[5,7,11]$. In this report, cytologic diagnoses obtained from fine-needle aspiration of granulomas can reveal characteristic giant cells with inflammatory cells. Severe inflammatory reactions may lead to difficulty in the clinical distinction of granulomas from tumors, because the giant cells that accumulate within such lesions often resemble tumor cells [2,6,12]. Diagnostic imaging offers the most useful information based on the specific characteristics. Potential radiographic findings of abdominal granulomas caused by gossypibomas were whirl-like gas patterns, focal calcifications, and intestinal obstruction [1,6]. Abdominal granulomas due to retained sutures could be diagnosed by homogenous soft tissue opacity on radiography $[4,5]$. These previously reported radiographic findings associated with suture granulomas were identical to those found in the three cases presented herein. The ultrasonographic characteristics of abdominal masses in the three cases presented in this report were a hyperechoic mass with no or slight acoustic shadowing. On ultrasonography, gossypibomas typically have as well-demarcated hypoechoic masses with highly echogenic, shadowing components [1,6]. Suture granulomas appear as variety of ultrasound findings such as a capsule-like mass with slight acoustic shadowing and a hyperechoic mass without acoustic shadowing [4,5]. No acoustic shadows may be seen in ultrasonographic images of pure abdominal granulomas without abscess formation, mineralization, or infection [6]. In addition, acoustic shadows may disappear as the lesion progresses based on diachronic changes on ultrasonographic views in human patients with retained surgical sponges [3]. The possible developmental process was shown by the initial formation of a capsule structure filled with purulent fluid surrounding the foreign material, and the following replacement of the granulomatous tissue that infiltrated into the foreign material [3]. The variability of the ultrasound findings may contribute to difficulty in diagnosis of abdominal granulomas.

In human patients, typical CT findings may also include a whirllike gas pattern as seen on radiography [1]. Additional CT findings may include enhancement of the rim of the mass after intravenous injection of contrast medium [1]. In veterinary field, to our knowledge, there was no report describing the CT findings of such lesions. The present cases had uniform contrast enhancement over all areas of the masses; the average Hus within the six abdominal masses in the three cases increased from 47.3 to 123.7 on postcontrast examination. This difference may be explained by either the development of pure granulomas without abscess formation or diachronic development of granulomas [6]. In the present report, the following characteristic CT findings of abdominal granulomas were seen: (1) irregular margin of the mass [12]; (2) uniformity of contrast enhancement over all areas of the mass; (3) and maintenance of lumen of the small intestine even if embedded by the mass. Clinical therapy can be chosen based on an accurate differential diagnosis for animals with multiple masses, as in our two cases, which is made possible by the combined use of CT with radiography, ultrasonography, blood examinations, and other tests as necessary.

\section{Conclusion}

The CT findings of abdominal granulomas were characterized by 1) masses with irregular margination, 2) uniformity of contrast enhancement over all areas of the mass; and 3) maintenance of lumen of the small intestine embedded by the mass. This can be helpful for diagnosis of abdominal granulomas in dogs.

\section{Conflict of Interest}

The authors declare that they have no conflict of interest. 


\section{References}

1. Merlo M, Lamb CR (2000) Radiographic and ultrasonographic features of retained surgical sponge in eight dogs. Vet Radiol Ultrasound 41: 279-283.

2. Putwain S, Archer J (2009) What is your diagnosis? Intra-abdominal mass aspirate from a spayed dog with abdominal pain. Vet Clin Pathol 38: 253-256.

3. Matsumoto S, Aikawa H, Miyake H, Mori H, Maeda T, et al. (1990) CT and sonographic diagnosis of retained surgical sponge. Nihon Igaku Hoshasen Gakkai Zasshi 50: 1350-1358.

4. Boza S, Lucas X, Zarelli M, Soler M, Belda E, et al. (2010) Late abscess formation caused by silk suture following hysterectomy in a female dog. Reprod Domest Anim 45: 934-936.

5. Yuki M, Hirano $T$ (2010) Use of a combination of prednisolone and rosuvastatin for treatment of a pyogranuloma in a dog. J Am Vet Med Assoc 236: 767-769.

6. Maï W, Ledieu D, Venturini L, Fournel C, Fau D, et al. (2001) Ultrasonographic appearance of intra-abdominal granuloma secondary to retained surgical sponge. Vet Radiol Ultrasound 42 : $157-160$.
7. Yamagishi C, Momoi Y, Kobayashi T, Ide K, Ohno K, et al. (2007) A retrospective study and gene analysis of canine sterile panniculitis. J Vet Med Sci 69: 915-924.

8. Pearson $H$ (1973) The complications of ovariohysterectomy in the bitch. J Small Anim Pract 14: 257-266.

9. Werner RE, Straughan AJ, Vezin D (1992) Nylon cable band reactions in ovariohysterectomized bitches. J Am Vet Med Assoc 200: 64-66.

10. Spackman CJA, Caywood DD, Johnston GR, Feeney DA (1984) Granulomas of the uterine and ovarian stumps: a case report. J Am Anim Hosp Assoc 20: 449-453.

11. Aikawa T, Yoshigae $Y$, Kanazono S (2008) Epidural idiopathic sterile pyogranulomatous inflammation causing spinal cord compressive injury in five Miniature Dachshunds. Vet Surg 37: 594-601.

12. Kim JH, Lee JH, Shong YK, Hong SJ, Ko MS, et al. (2009) Ultrasound features of suture granulomas in the thyroid bed after thyroidectomy for papillary thyroid carcinoma with an emphasis on their differentiation from locally recurrent thyroid carcinomas. Ultrasound Med Biol 35: 1452-1457. 